\title{
Commentary: Surgical outcome for infective endocarditis in children during long term follow-up
}

Koh Takeuchi, MD, PhD

\author{
From the Department of Cardiovascular Surgery, Hanyu General Hospital, Hanyu, Japan \\ Disclosures: Author has nothing to disclose with regard to commercial support. \\ Received for publication July 25, 2019; accepted for publication July 29, 2019. \\ Address for reprints: Koh Takeuchi, MD, PhD, Department of Cardiovascular Surgery, Hanyu General Hospital, \\ 446 Shimoiwase, Hanyu, Saitama, 348-8505 Japan (E-mail: koutakeuchi-circ@umin.ac.jp). \\ J Thorac Cardiovasc Surg 2019;158:1411-2 \\ $0022-5223 / \$ 36.00$ \\ Copyright $(2019$ by The American Association for Thoracic Surgery \\ https://doi.org/10.1016/j.jtcvs.2019.07.113
}

Infective endocarditis (IE) has been increased in the adult population because of an increase of the number of drug users. In children, there has been an increase in cases of IE associated with congenital heart disease (CHD), because most patients with CHD survive much longer than they did several decades ago. Early surgical correction of lesions that were major risk factors for IE in the past has also changed the substrate for this disease. Increasingly, IE is seen to develop in the absence of CHD. This circumstance is often associated with central indwelling venous catheters (central lines). Many important clinical questions addressed in the guidelines do not lend themselves to clinical trials. It has been reported that in the case of IE in the structurally normal heart, the infection usually involves the aortic or mitral valve secondary to Staphylococcus aureus bacteremia. ${ }^{1}$ Because a large study may be difficult to conduct in the pediatric population, current recommendations for treatment of the pediatric population with IE are extrapolated from the guidelines for adults.

In this issue of the Journal, Khoo and associates ${ }^{2}$ describe the surgical outcomes of children with IE in a single institution. One hundred thirty-eight patients were involved in this study, including infants $(20 \%$ of all patients with IE). Of these, 10 patients had univentricular circulation and 128 patients had biventricular circulation. This includes $43.5 \%$ of patients with left-sided IE and $42.7 \%$ of patients with right-sided IE, with the remaining $6.5 \%$ of patients having lesions on both sides. Valvular IE lesions were seen in $65.2 \%$ of the patients with a native valve IE and $20.3 \%$ of patients with a prosthetic valve IE. Nonvalvular IE was seen in $14.4 \%$ of all patients. Relative to a previous study on culture-negative $\mathrm{IE}^{3}{ }^{3}$ the blood culture-positive rate in the study of Khoo and associates ${ }^{2}$ was relatively high $(79 \%)$. Among those 109 patients, gram-positive infection was more frequent $(85.3 \%)$ than gram-negative infection (14.4\%). S aureus was the most commonly found organism $(25.3 \%)$, whereas coagulase-negative staphylococcus had a stronger impact on mortality or morbidity, a finding consistent with adult data. ${ }^{4}$ In $31.2 \%$ of patients,

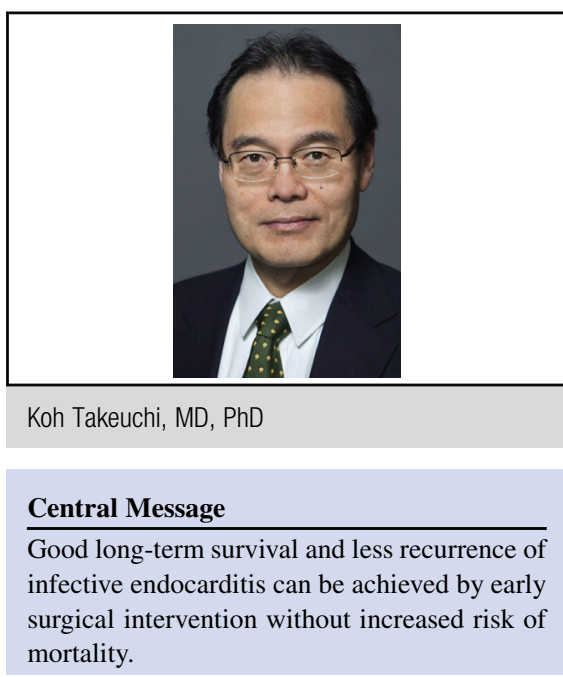

See Article page 1399.

the antibiotic regimen had to be changed during the treatment. The median times for an embolic event from the date of the surgery were 5 days in patients with left-sided IE, and 28 days in patients with right sided IE. Aortic valve IE, which was the most frequent form of IE, carried a higher operative mortality $(12.8 \%)$. Khoo and associates ${ }^{2}$ showed higher mortality in left-sided IE, which was similar to the findings of a previous report. ${ }^{5}$ Early surgery was safe and did not increase the risk of mortality. Catheter-associated infections occurred in $77 \%$ of children younger than 10 years of age. Younger children were more likely to undergo surgery while still receiving antibiotics. Coagulase-negative staphylococcus is becoming the most common infectious organism. ${ }^{6}$ It has been recognized that coagulase-negative staphylococcus causes increased morbidity and mortality in the case of prosthetic valve IE. It would seem reasonable to recommend early surgical intervention, because freedom from recurrence of IE was $95 \%$ at a 25 years of follow-up, suggesting that surgery is highly effective in curing IE in children. The reoperation rate not directly related to IE was also high $(45 \%$ of patients requiring reoperation by 25 years), particularly in patients with preexisting heart defects and perivalvular abscesses.

Although there are several limitations, including time bias, which may have allowed a change in antibiotic regimen and surgical indications, wide age distribution, and selection bias (surgical case only), the report of Khoo and associates ${ }^{2}$ provides useful information about pediatric 
patients with IE. The results with regard to causative organisms, the location of IE, and the severity of IE were similar to those seen in adult populations. Early surgical intervention is recommended in left-sided IE, particularly in aortic valve endocarditis. A strategy to reduce reoperation not directly related to IE may be a key for success in the future.

\section{References}

1. Baltimore RS, Gewitz M, Baddour LM, Beerman LB, Jackson MA, Lockhart PB, et al; American Heart Association Rheumatic Fever, Endocarditis, and Kawasaki Disease Committee of the Council on Cardiovascular Disease in the Young and the Council on Cardiovascular and Stroke Nursing. Infective endocarditis in childhood: 2015 update: a scientific statement from the American Heart Association. Circulation. 2015;132:1487-515.
2. Khoo B, Buratto E, Fricke TA, Gelbart B, Brizard CP, Brink KJ, et al. Outcome of surgery for infective endocarditis in childrens: a 30-year experience. J Thorac Cardiovasc Surg. 2019;158:1399-409.

3. Kupferwasser I, Darius H, Müller A, Martin C, Mohr-Kahaly S, Erbel R, et al. Diagnosis of culture negative endocarditis: the role of the Duke criteria and the impact of transesophageal echocardiography. Am Heart J. 2001;142:146-52.

4. Chu VH, Miro JM, Hoen B, Cabell CH, Pappas PA, Jones P, et al; International Collaboration on Endocarditis-Prospective Cohort Study Group. Coagulase-negative staphylococcal prosthetic valve endocarditis - a contemporary update based on the International Collaboration on Endocarditis: prospective cohort study. Heart. 2009;95:570-6.

5. Shamszad P, Khan MS, Rossano JW, Fraser CD Jr. Early surgical therapy of infective endocarditis in children: a 15-year experience. J Thorac Cardiovasc Surg. 2013;146:506-11.

6. Tseng WC, Chiu SN, Shao PL, Wang JK, Chen CA, Lin MT, et al. Changing spectrum of infective endocarditis in children: a 30 years experiences from a tertiary care center in Taiwan. Pediatr Infect Dis J. 2014;33:467-71. 